\title{
MANIFOLDS COVERED BY LINES AND EXTREMAL RAYS
}

\author{
CARLA NOVELLI AND GIANLUCA OCCHETTA
}

\begin{abstract}
Let $X$ be a smooth complex projective variety and let $H \in \operatorname{Pic}(X)$ be an ample line bundle. Assume that $X$ is covered by rational curves with degree one with respect to $H$ and with anticanonical degree greater than or equal to $(\operatorname{dim} X-1) / 2$. We prove that there is a covering family of such curves whose numerical class spans an extremal ray in the cone of curves $\mathrm{NE}(X)$.
\end{abstract}

\section{INTRODUCTION}

Let $X$ be a smooth complex projective variety which admits a morphism with connected fibers $\varphi: X \rightarrow Z$ onto a normal variety $Z$ such that the anticanonical bundle $-K_{X}$ is $\varphi$-ample, $\operatorname{dim} X>\operatorname{dim} Z$ and $\rho_{X}=\rho_{Z}+1$ (i.e. an elementary extremal contraction of fiber type).

It is well known, by fundamental results of Mori theory, that through every point of $X$ there is a rational curve contracted by $\varphi$. The numerical classes of these curves lie in an extremal ray of the cone $\mathrm{NE}(X)$. By taking a covering family of such curves one obtains a quasi-unsplit family of rational curves, i.e. a family such that the irreducible components of all the degenerations of curves in the family are numerically proportional to a curve in the family. It is very natural to ask if the converse is also true:

Given a covering quasi-unsplit family $V$ of rational curves, is there an extremal elementary contraction which contracts all curves in the family or, in other words, does the numerical class of a curve in the family span an extremal ray of $\mathrm{NE}(X)$ ?

As proved in [8] (see also [10] and [14]) there is always a rational fibration, defined on an open set of $X$, whose general fibers are proper, which contracts a general curve in $V$. More precisely, a general fiber is an equivalence class with respect to the relation induced by the closure $\mathcal{V}$ of the family $V$ in the Chow scheme of $X$ in the following way: two points $x$ and $y$ are equivalent if there exists a connected chain of cycles in $\mathcal{V}$ which joins $x$ and $y$.

By a careful study of this fibration and of its indeterminacy locus, a partial answer to this question has been given in [6. Theorem 2]; namely, if the dimension of a general equivalence class is greater than or equal to the dimension of the variety minus three then the numerical class of a general curve in the family spans an extremal ray of $\mathrm{NE}(X)$.

Before the results in $[\underline{6}$ a special but very natural situation in which the question arises has been studied in [5]. In that paper manifolds covered by rational curves

2010 Mathematics Subject Classification. 14J40, 14E30, 14 C99.

Key words and phrases. Rational curves, extremal rays. 
of degree one with respect to an ample line bundle $H$ were considered, and it was proved that a covering family of such curves (we will call them lines, by abuse) of anticanonical degree greater than or equal to $\frac{\operatorname{dim} X+2}{2}$ spans an extremal ray (see also [4, Theorem 2.4]).

Recently, in 15, Theorem 7.3], the extremality of a covering family $V$ of lines was proved under the weaker assumption that the anticanonical degree of such curves, denoted by abuse of notation by $-K_{X} \cdot V$, is greater than or equal to $\frac{\operatorname{dim} X+1}{2}$.

The goal of the present paper is to prove the following

Theorem. Let $(X, H)$ be a polarized manifold with a dominating family of rational curves $V$ such that $H \cdot V=1$. If $-K_{X} \cdot V \geq \frac{\operatorname{dim} X-1}{2}$, then $[V]$ spans an extremal ray of $\mathrm{NE}(X)$.

The main idea is, as in [15], to combine the ideas and tecniques of [5], especially taking into consideration a suitable adjoint divisor $K_{X}+m H$ and studying its nefness, with those of [6, in particular regarding the existence of special curves in the indeterminacy locus of the rational fibration associated to $V$.

\section{BACKGROUND MATERIAL}

Let $X$ be a smooth projective variety defined over the field of complex numbers. A contraction $\varphi: X \rightarrow Z$ is a proper surjective map with connected fibers onto a normal variety $Z$.

If the canonical bundle $K_{X}$ is not nef, then the negative part of the cone $\mathrm{NE}(X)$ of effective 1-cycles is locally polyhedral, by the Cone Theorem. By the Contraction Theorem, to every face in this part of the cone is associated a contraction.

Unless otherwise stated, we will reserve the name extremal face for a face contained in $\overline{\mathrm{NE}}(X) \cap\left\{a \in \mathrm{N}_{1}(X) \mid K_{X} \cdot a<0\right\}$, and we will call extremal contraction the contraction of such a face.

An extremal contraction associated to an extremal face of dimension one, i.e. to an extremal ray, is called an elementary contraction; an extremal ray $\tau$ is called numerically effective, and the associated contraction is said to be of fiber type, if $\operatorname{dim} Z<\operatorname{dim} X$; otherwise the ray is called non nef and the contraction is birational. If the codimension of the exceptional locus of an elementary birational contraction is equal to one, the ray and the contraction are called divisorial, otherwise they are called small.

A Cartier divisor which is the pull-back of an ample divisor $A$ on $Z$ is called a supporting divisor of the contraction $\varphi$.

If the anticanonical bundle of $X$ is ample, $X$ is called a Fano manifold. For a Fano manifold, the index, denoted by $r_{X}$, is defined as the largest natural number $r$ such that $-K_{X}=r H$ for some (ample) divisor $H$ on $X$.

Throughout the paper, unless otherwise stated, we will use the word curve to denote an irreducible curve.

Definition 1.1. A family of rational curves is an irreducible component $V \subset$ $\operatorname{Ratcurves}^{n}(X)$ (see [14, Definition 2.11]). Given a rational curve we will call a family of deformations of that curve any irreducible component of $\operatorname{Ratcurves}^{n}(X)$ containing the point parametrizing that curve. We will say that $V$ is unsplit if it is proper. We define $\operatorname{Locus}(V)$ to be the set of points of $X$ through which there is a curve among those parametrized by $V$; we say that $V$ is a covering family if 
$\operatorname{Locus}(V)=X$ and that $V$ is a dominating family if $\overline{\operatorname{Locus}(V)}=X$.

We denote by $V_{x}$ the subscheme of $V$ parametrizing rational curves passing through $x \in \operatorname{Locus}(V)$ and by $\operatorname{Locus}\left(V_{x}\right)$ the set of points of $X$ through which there is a curve among those parametrized by $V_{x}$.

By abuse of notation, given a line bundle $L \in \operatorname{Pic}(X)$, we will denote by $L \cdot V$ the intersection number $L \cdot C_{V}$, with $C_{V}$ any curve among those parametrized by $V$.

Proposition 1.2. ([14, IV.2.6]) Let $V$ be an unsplit family of rational curves on $X$. Then

(a) $\operatorname{dim} \operatorname{Locus}(V)+\operatorname{dim} \operatorname{Locus}\left(V_{x}\right) \geq \operatorname{dim} X-K_{X} \cdot V-1$;

(b) every irreducible component of $\operatorname{Locus}\left(V_{x}\right)$ has dimension $\geq-K_{X} \cdot V-1$.

This last proposition, in case $V$ is the unsplit family of deformations of a rational curve of minimal anticanonical degree in an extremal face of $\mathrm{NE}(X)$, gives the fiber locus inequality:

Proposition 1.3. ([12, Theorem 0.4], [19, Theorem 1.1]) Let $\varphi$ be a Fano-Mori contraction of $X$. Denote by $E$ the exceptional locus of $\varphi$ and by $F$ an irreducible component of a non-trivial fiber of $\varphi$. Then

$$
\operatorname{dim} E+\operatorname{dim} F \geq \operatorname{dim} X+\ell-1,
$$

where $\ell:=\min \left\{-K_{X} \cdot C \mid C\right.$ is a rational curve in $\left.F\right\}$. If $\varphi$ is the contraction of an extremal ray $\tau$, then $\ell(\tau):=\ell$ is called the length of the ray.

Definition 1.4. We define a Chow family of rational curves $\mathcal{W}$ to be an irreducible component of $\operatorname{Chow}(X)$ parametrizing rational and connected 1-cycles.

We define $\operatorname{Locus}(\mathcal{W})$ to be the set of points of $X$ through which there is a cycle among those parametrized by $\mathcal{W}$; notice that $\operatorname{Locus}(\mathcal{W})$ is a closed subset of $X$ (14, II.2.3]). We say that $\mathcal{W}$ is a covering family if $\operatorname{Locus}(\mathcal{W})=X$.

Definition 1.5. If $V$ is a family of rational curves, the closure of the image of $V$ in $\operatorname{Chow}(X)$, denoted by $\mathcal{V}$, is called the Chow family associated to $V$.

Remark 1.6. If $V$ is proper, i.e. if the family is unsplit, then $V$ corresponds to the normalization of the associated Chow family $\mathcal{V}$.

Definition 1.7. Let $\mathcal{V}$ be the Chow family associated to a family of rational curves $V$. We say that $V$ (and also $\mathcal{V}$ ) is quasi-unsplit if every component of any reducible cycle in $\mathcal{V}$ is numerically proportional to $V$.

Definition 1.8. Let $\mathcal{W}$ be a Chow family of rational curves on $X$ and $Z \subset X$. We define $\operatorname{Locus}(\mathcal{W})_{Z}$ to be the set of points $x \in X$ such that there exists a cycle $\Gamma$ among those parametrized by $\mathcal{W}$ with $\Gamma \cap Z \neq \emptyset$ and $x \in \Gamma$.

We define $\operatorname{ChLocus}(\mathcal{W})_{Z}$ to be the set of points $x \in X$ such that there exists a chain of cycles among those parametrized by $\mathcal{W}$ connecting $x$ and $Z$. Notice that, a priori $\operatorname{ChLocus}(\mathcal{W})_{Z}$ is a countable union of closed subsets of $X$.

Notation: If $T \subset X$ we will denote by $\mathrm{N}_{1}(T, X) \subset \mathrm{N}_{1}(X)$ the vector subspace generated by numerical classes of curves in $T$; we will denote by $\mathrm{NE}(T, X) \subset \mathrm{NE}(X)$ the subcone generated by numerical classes of curves in $T$.

The notation $\langle\ldots\rangle$ will denote a linear subspace, while the notation $\langle\ldots\rangle_{c}$ will denote a subcone. 
Lemma 1.9. (14, Proposition IV.3.13.3], 1, Lemma 4.1]) Let $T \subset X$ be a closed subset and let $\mathcal{W}$ be a Chow family of rational curves. Then every curve contained in $\operatorname{ChLocus}(\mathcal{W})_{T}$ is numerically equivalent to a linear combination with rational coefficients of a curve contained in $T$ and irreducible components of cycles among those parametrized by $\mathcal{W}$ which intersect $T$.

Lemma 1.10. (Cf. [5, Proof of Lemma 1.4.5], 17, Lemma 1]) Let $T \subset X$ be a closed subset and let $V$ be a quasi-unsplit family of rational curves. Then every curve contained in $\operatorname{ChLocus}(\mathcal{V})_{T}$ is numerically equivalent to a linear combination with rational coefficients

$$
\lambda C_{T}+\mu C_{V}
$$

where $C_{T}$ is a curve in $T, C_{V}$ is a curve among those parametrized by $V$ and $\lambda \geq 0$.

Corollary 1.11. (Cf. 9, Corollary 2.2 and Remark 2.4]) Let $\Sigma$ be an extremal face of $\mathrm{NE}(X)$ and denote by $F$ a fiber of the contraction associated to $\Sigma$. Let $V$ be a quasi-unsplit family numerically independent from curves whose numerical class is in $\Sigma$. Then

$$
\operatorname{NE}\left(\operatorname{ChLocus}(\mathcal{V})_{F}, X\right)=\langle\Sigma,[V]\rangle_{c},
$$

i.e. the numerical class in $X$ of a curve in $\operatorname{ChLocus}(\mathcal{V})_{F}$ is in the subcone of $\mathrm{NE}(X)$ generated by $\Sigma$ and $[V]$.

Lemma 1.12. Let $D$ be an effective divisor on $X$ and $L$ a nef divisor. If $\left.(L+D)\right|_{D}$ is nef then $L+D$ is nef.

Proof. Assume that $\gamma$ is an effective curve on $X$ such that $(L+D) \cdot \gamma<0$. By the nefness of $L$ we have $D \cdot \gamma<0$, hence $\gamma \subset D$. But $L+D$ is nef on $D$, a contradiction.

\section{Rationally CONNECTED FibRations}

Let $X$ be a smooth complex projective variety and let $\mathcal{W}$ be a covering Chow family of rational curves.

Definition 2.1. The family $\mathcal{W}$ defines a relation of rational connectedness with respect to $\mathcal{W}$, which we shall call $r c(\mathcal{W})$-relation for short, in the following way: $x$ and $y$ are in $\operatorname{rc}(\mathcal{W})$-relation if there exists a chain of cycles among those parametrized by $\mathcal{W}$ which joins $x$ and $y$.

To the $\operatorname{rc}(\mathcal{W})$-relation we can associate a fibration, at least on an open subset ([7, [14, IV.4.16]); we will call it $r c(\mathcal{W})$-fibration.

In the notation of [6], by [10, Theorem 5.9] there exists a closed irreducible subset of $\operatorname{Chow}(X)$ such that, denoting by $Y$ its normalization and by $Z \subset Y \times X$ the restriction of the universal family, we have a commutative diagram

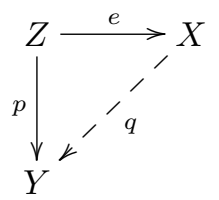

where $p$ is the projection onto the first factor and $e$ is a birational morphism whose exceptional locus $E$ does not dominate $Y$. Moreover, a general fiber of $q$ is irreducible and is $\operatorname{arc}(\mathcal{W})$-equivalence class.

Let $B$ be the image of $E$ in $X$; note that $\operatorname{dim} B \leq \operatorname{dim} X-2$, as $X$ is smooth. 
If we consider a (covering) Chow family $\mathcal{V}$, associated to a quasi-unsplit dominating family $V$, then by [6, Proposition $1,(\mathrm{ii})] B$ is the union of all $\operatorname{rc}(\mathcal{V})$-equivalence classes of dimension greater than $\operatorname{dim} X-\operatorname{dim} Y$.

Moreover we have the following

Lemma 2.2. Let $V$ be a quasi-unsplit dominating family of rational curves on a smooth complex projective variety $X$. Let $B$ be the indeterminacy locus of the $r c(\mathcal{V})$-fibration $q: X--\rightarrow Y$, let $D$ be a very ample divisor on $q(X \backslash B)$ and let $\widehat{D}:=\overline{q^{-1} D}$. Then

(1) $\widehat{D} \cdot V=0$;

(2) if $C \not \subset B$ is a curve not numerically proportional to $[V]$, then $\widehat{D} \cdot C>0$;

(3) if $\widehat{D} \cdot C>0$ for every curve $C \subset B$ not numerically proportional to $[V]$, then $[V]$ spans an extremal ray of $\mathrm{NE}(X)$.

Proof. See [6, Proof of Proposition 1].

Corollary 2.3. [6, Proposition 3]. Let $V$ be a quasi-unsplit dominating family of rational curves on a smooth complex projective variety $X$; denote by $B$ the indeterminacy locus of the $r c(\mathcal{V})$-fibration and by $f_{V}$ the dimension of the general $r c(\mathcal{V})$-equivalence class.

If $[V]$ does not span an extremal ray of $\mathrm{NE}(X)$, then $B$ is not empty. In particular there exist $r c(\mathcal{V})$-equivalence classes of dimension $\geq f_{V}+1$.

We now give a lower bound on the dimension of $\operatorname{ChLocus}(\mathcal{V})_{S}$, depending on the position of the subvariety $S$ with respect to the indeterminacy locus of the $\operatorname{rc}(\mathcal{V})$-fibration.

Lemma 2.4. Let $V$ be a quasi-unsplit dominating family of rational curves on a smooth complex projective variety $X$; denote by $B$ the indeterminacy locus of the $r c(\mathcal{V})$-fibration and by $f_{V}$ the dimension of the general $r c(\mathcal{V})$-equivalence class.

Let $S \subset X$ be an irreducible subvariety such that $[V] \notin \mathrm{NE}(S, X)$. Then there exists an irreducible $X_{S}$ contained in $\operatorname{ChLocus}(\mathcal{V})_{S}$ such that

(1) if $S \not \subset B$, then $\operatorname{dim} X_{S} \geq \operatorname{dim} S+f_{V}$;

(2) if $S \subset B$, then $\operatorname{dim} X_{S} \geq \operatorname{dim} S+f_{V}+1$.

Moreover, $X_{S}$ is not $r c(\mathcal{V})$-connected.

Proof. We refer to diagram 2.1.1). Given any $T \subset Z$ we will set $Z_{T}:=p^{-1}(p(T))$. Let $S^{\prime} \subset Z$ be an irreducible component of $e^{-1}(S)$ which dominates $S$ via $e$.

By our assumptions on $\mathrm{NE}(S, X)$ we have that $S^{\prime}$ meets any fiber of $\left.p\right|_{Z_{S^{\prime}}}$ in points so, up to replace $Z_{S^{\prime}}$ with $S^{\prime} \times_{p\left(S^{\prime}\right)} Z_{S^{\prime}}$, we can assume that $S^{\prime}$ is a section of $\left.p\right|_{Z_{S^{\prime}}}$. Let $Z^{\prime}$ be an irreducible component of $Z_{S^{\prime}}$ which contains $S^{\prime}$. We have

$$
\operatorname{dim} Z^{\prime} \geq \operatorname{dim} p\left(S^{\prime}\right)+f_{V}=\operatorname{dim} S^{\prime}+f_{V} \geq \operatorname{dim} S+f_{V} .
$$

Moreover, notice that $S=e\left(S^{\prime}\right) \subset e\left(Z^{\prime}\right) \subset e\left(Z_{S^{\prime}}\right) \subset \operatorname{ChLocus}(\mathcal{V})_{S}$.

Assume that $S \not \subset B$. Then $Z^{\prime} \not \subset E$, hence the map $\left.e\right|_{Z^{\prime}}: Z^{\prime} \rightarrow X$ is generically finite. Therefore, in view of 2.4.1), $\operatorname{dim} e\left(Z^{\prime}\right)=\operatorname{dim} Z^{\prime} \geq \operatorname{dim} S+f_{V}$; moreover, since $S \subset e\left(Z^{\prime}\right)$ we have that $e\left(Z^{\prime}\right)$ is not $\operatorname{rc}(\mathcal{V})$-connected.

Assume now that $S \subset B$. Assertion (2) will follow once we prove that the general fiber $G$ of $\left.e\right|_{Z}$ has dimension strictly smaller than the general fiber of $\left.e\right|_{S^{\prime}}$ for at 
least one irreducible component $\bar{Z}$ of $Z_{S^{\prime}}$ which dominates $p\left(S^{\prime}\right)$. In fact, recalling also 2.4.1), in this case we will have

$\operatorname{dim} e(\bar{Z})=\operatorname{dim} \bar{Z}-\operatorname{dim} G>\left(\operatorname{dim} S^{\prime}+f_{V}\right)-\left(\operatorname{dim} S^{\prime}-\operatorname{dim} S\right)=f_{V}+\operatorname{dim} S$.

Claim. Let $G$ be an irreducible component of a fiber of $\left.e\right|_{Z_{S^{\prime}}}$, let $z \in G$ be any point and let $z^{\prime}:=p^{-1}(p(z)) \cap S^{\prime}$ be the intersection of the fiber of $p$ containing $z$ with $S^{\prime}$; then there exists an irreducible component $F$ of the fiber $F^{\prime}$ of $\left.e\right|_{S^{\prime}}$ containing $z^{\prime}$ such that $p(G) \subseteq p(F)$.

To prove the claim, recall that, since $e\left(Z_{G}\right) \subset \operatorname{ChLocus}(\mathcal{V})_{e(z)}$, the image via $e$ of any curve in $Z_{G} \cap S^{\prime}$ - which is irreducible, being a section over $p(G)$ - must be a point, otherwise it would be a curve contained in $S \cap \operatorname{ChLocus}(\mathcal{V})_{e(z)}$, which is a contradiction, since curves in $S$ are numerically independent from $[V]$.

Therefore $Z_{G} \cap S^{\prime}$ is contained in a fiber $F^{\prime}$ of $\left.e\right|_{S^{\prime}}$. To prove the claim we take as $F$ the irreducible component of $F^{\prime}$ containing $Z_{G} \cap S^{\prime}$.

Let $S^{1} \subset S^{\prime}$ be the proper closed subset on which $\left.e\right|_{S^{\prime}}$ is not equidimensional and let $S^{2} \subset S^{\prime}$ be the proper closed subset of points in which the fiber of $\left.e\right|_{S^{\prime}}$ is not locally irreducible. Recalling that $\left.p\right|_{S^{\prime}}$ is a finite map we see that $p\left(S^{1} \cup S^{2}\right)$ is a proper closed subset of $p\left(S^{\prime}\right)$.

Let $y \in p\left(S^{\prime}\right) \backslash p\left(S^{1} \cup S^{2}\right)$ be a general point; in particular there is only one irreducible component $F$ of the fiber $F^{\prime}$ of $\left.e\right|_{S^{\prime}}$ passing through $z^{\prime}=p^{-1}(y) \cap S^{\prime}$ and $\operatorname{dim} F=\operatorname{dim} S^{\prime}-\operatorname{dim} S$.

Notice that $\operatorname{dim} e\left(Z_{F}\right)>f_{V}$, otherwise a one parameter family of fibers of $p$ meeting $F$ would have the same image in $X$ (Cf. [6, End of proof of Proposition 1], where $\left.e\left(Z_{F}\right)=\operatorname{Locus}\left(V_{e(F)}\right)\right)$.

This implies that, for an irreducible component $\bar{Z}_{F}$ of $Z_{F}$ we have $\operatorname{dim} e\left(\bar{Z}_{F}\right)>f_{V}$. Taking as $\bar{Z}$ an irreducible component of $Z_{S^{\prime}}$ containing $\bar{Z}_{F}$ we have that, for every point $z \in p^{-1}(y) \cap \bar{Z}$ and any irreducible component $G$ of the fiber of $\left.e\right|_{\bar{Z}}$ passing through $z$ we have $p(G) \subseteq p(F)$, hence $\operatorname{dim} G<\operatorname{dim} F=\operatorname{dim} S^{\prime}-\operatorname{dim} S$; the same inequality then holds for the general fiber by semicontinuity of the local dimension. Noticing that $S$ is contained in $\operatorname{ChLocus}_{e}(\bar{Z})(\mathcal{V})$ the last assertion follows.

Remark 2.5. Both the bounds in Lemma 2.4 are sharp. An example for the second one is given by [6. Example 2]: in that example $B \simeq \mathbb{P}^{2} \times \mathbb{P}^{1}$; taking as $S$ a fiber of the projection onto $\mathbb{P}^{2}$ we have equality in (2).

\section{BLOWING-DOWN}

In this section we consider the following situation, which will show up in the proof of Theorem 4.3):

Lemma 3.1. Let $(X, H)$ be a polarized manifold with a dominating family of rational curves $V$ such that $H \cdot V=1$. Denote by $f_{V}$ the dimension of the general $r c(\mathcal{V})$-equivalence class and assume that there exists an extremal face $\Sigma$ in $\mathrm{NE}(X)$ whose associate contraction $\sigma: X \rightarrow X^{\prime}$ is a smooth blow-up along a disjoint union of subvarieties $T_{i}$ of dimension $\leq f_{V}$ such that $E_{i} \cdot V=0$ for every exceptional divisor $E_{i}$ and $H \cdot l_{i}=1$ if $l_{i}$ is a line in a fiber of $\sigma$. Finally denote by $V^{\prime}$ a family of deformation of $\sigma(C)$, with $C$ a general curve parametrized by $V$. Then

(1) $-K_{X^{\prime}} \cdot V^{\prime}=-K_{X} \cdot V$;

(2) there exists an ample line bundle $H^{\prime}$ on $X^{\prime}$ such that $H^{\prime} \cdot V^{\prime}=1$; 
(3) if $C^{\prime}$ is a curve parametrized by $V^{\prime}$ such that $T_{i} \cap C^{\prime} \neq \emptyset$, then $C^{\prime} \subset T_{i}$;

(4) $\rho_{X^{\prime}}>1$;

(5) if $\left[V^{\prime}\right]$ spans an extremal ray of $\mathrm{NE}\left(X^{\prime}\right)$, then $[V]$ spans an extremal ray of $\mathrm{NE}(X)$.

Proof. It is enough to prove the statement in case $\operatorname{dim} \Sigma=1$, i.e. $\sigma: X \rightarrow X^{\prime}$ is the blow-up of $X^{\prime}$ along a smooth subvariety $T$ associated to the extremal ray $\Sigma$. In fact, if $\operatorname{dim} \Sigma>1$, the contraction of $\Sigma$ factors through elementary contractions, each one satisfying the assumptions in the statement.

Denote by $E$ the exceptional locus of $\sigma$. Since $E \cdot V=0$ the first assertion in the statement follows from the canonical bundle formula for blow-ups.

Moreover, the fact that $E \cdot V=0$ also implies that any $\operatorname{rc}(\mathcal{V})$-equivalence class meeting $E$ is actually contained in $E$. Therefore, if $F$ is a non-trivial fiber of $\sigma$, then $\operatorname{ChLocus}(\mathcal{V})_{F} \subseteq E$. By Lemma 2.4

$$
\operatorname{dim} \operatorname{ChLocus}(\mathcal{V})_{F} \geq f_{V}+\operatorname{dim} F \geq \operatorname{dim} X-1,
$$

hence $E=\operatorname{ChLocus}(\mathcal{V})_{F}$ and $\operatorname{dim} T=f_{V}$; in particular, applying Corollary 1.11 we get that $\mathrm{NE}(E, X)=\langle[V], \Sigma\rangle_{c}$.

The line bundle $\left.(H+E)\right|_{E}$ is nef and it is trivial only on $\Sigma$, since $(H+E) \cdot \Sigma=0$ and $(H+E) \cdot V=1$. Then $H+E$ is nef by Lemma 1.12 .

Notice also that $H+E$ is trivial only on $\Sigma$. Indeed, let $\gamma$ be an effective curve on $X$ such that $(H+E) \cdot \gamma=0$. Due to the ampleness of $H$ we have $E \cdot \gamma<0$, hence $\gamma \subset E$. This implies that $[\gamma] \in \Sigma$. Therefore $H+E=\sigma^{*} H^{\prime}$, with $H^{\prime}$ an ample line bundle on $X^{\prime}$. By the projection formula $H^{\prime} \cdot V^{\prime}=1$, hence part (2) in the statement is proved.

Now, let $C^{\prime}$ be a curve parametrized by $V^{\prime}$ meeting $T$ and assume by contradiction that $C^{\prime}$ is not contained in $T$; denote by $\widetilde{C}^{\prime}$ its strict transform. Then

$$
1=H^{\prime} \cdot C^{\prime}=\sigma^{*} H^{\prime} \cdot \widetilde{C}^{\prime}=(H+E) \cdot \widetilde{C}^{\prime} \geq 2,
$$

which is a contradiction. It follows that every curve parametrized by $V^{\prime}$ which meets $T$ is contained in it; so we get part (3) in the statement.

As to part (4), assume by contradiction that $\rho_{X^{\prime}}=1$. This implies that $X^{\prime}$ is $\operatorname{rc}\left(\mathcal{V}^{\prime}\right)$-connected, but this is impossible as, in view of part (3), we cannot join points of $T$ and points outside of $T$ with curves parametrized by $V^{\prime}$.

Finally, to prove part (5) assume that $\left[V^{\prime}\right]$ spans an extremal ray of $X^{\prime}$ and let $B$ be the indeterminacy locus of the $\operatorname{rc}(\mathcal{V})$-fibration. We claim that $E \cap B=\emptyset$.

Assume by contradiction that this is not the case; then $E$ meets (and hence contains) an $\operatorname{rc}(\mathcal{V})$-equivalence class $G$ of dimension $\operatorname{dim} G \geq f_{V}+1$. Take a fiber $F$ of $\sigma$ meeting $G$. Then $\operatorname{dim} F+\operatorname{dim} G>\operatorname{dim} E$. On the other hand, $\operatorname{dim}(F \cap G)=0$ as $[V] \notin \Sigma$. So we get a contradiction.

Let $A$ be a supporting divisor of the contraction associated to $\left[V^{\prime}\right]$. The pullback $\sigma^{*} A$ defines a two-dimensional face $\Pi$ of $\overline{\mathrm{NE}}(X)$ containing $\Sigma$ and $[V]$. Let $\widehat{D}$ be as in Lemma 2.2 ; by the same lemma $\widehat{D} \cdot \Sigma>0$ and $\widehat{D} \cdot V=0$.

Assume that $\Pi$ is not spanned by $\Sigma$ and $[V]$; in this case there exists a class $c \in \overline{\mathrm{NE}}(X)$ belonging to $\Pi$ such that $E \cdot c>0$ and $\widehat{D} \cdot c<0$.

Let $\left\{C_{n}\right\}$ be a sequence of effective one cycles such that the limit of $\mathbb{R}_{+}\left[C_{n}\right]$ is $\mathbb{R}_{+} c$; 
by continuity, for some $n_{0}$ we have $E \cdot C_{n}>0$ and $\widehat{D} \cdot C_{n}<0$ for $n \geq n_{0}$, hence $C_{n} \subset B$, and $E \cap C_{n} \neq \emptyset$ for $n \geq n_{0}$, contradicting $E \cap B=\emptyset$.

\section{MAIN THEOREM}

First of all we consider polarized manifolds $(X, H)$ with a quasi-unsplit dominating family of rational curves $V$ proving that if, for $m$ large enough, the adjoint divisor $K_{X}+m H$ defines an extremal face containing $[V]$, then $[V]$ spans an extremal ray of $X$.

Proposition 4.1. Let $(X, H)$ be a polarized manifold which admits a quasi-unsplit dominating family of rational curves $V$; denote by $f_{V}$ the dimension of a general $r c(\mathcal{V})$-equivalence class.

If, for some integer $m$ such that $m+f_{V} \geq \operatorname{dim} X-3$, the divisor $K_{X}+m H$ is nef and it is trivial on $[V]$, then $[V]$ spans an extremal ray of $\mathrm{NE}(X)$.

Proof. Assume by contradiction that $[V]$ does not span an extremal ray in $\mathrm{NE}(X)$. This implies that $K_{X}+m H$ defines an extremal face $\Sigma$ of dimension at least two, containing $[V]$. By [15, Lemma 7.2] there exists an extremal ray $\vartheta \in \Sigma$ whose exceptional locus is contained in the indeterminacy locus $B$ of the $\operatorname{rc}(\mathcal{V})$-fibration. Since $\left(K_{X}+m H\right) \cdot \vartheta=0$, the length $\ell(\vartheta)$ is greater than or equal to $m$.

Let $F$ be a non-trivial fiber of the contraction associated to $\vartheta$; since this contraction is small, being $\operatorname{dim} B \leq \operatorname{dim} X-2$, then $\operatorname{dim} F \geq m+1$ by Proposition (1.3).

By part (2) of Lemma (2.4), the dimension of $\operatorname{ChLocus}(\mathcal{V})_{F}$ is

$$
\operatorname{dim} \operatorname{ChLocus}(\mathcal{V})_{F} \geq \operatorname{dim} F+f_{V}+1 .
$$

As the $\operatorname{rc}(\mathcal{V})$-equivalence classes are either contained in $B$ or have empty intersection with it, $\operatorname{ChLocus}(\mathcal{V})_{F} \subset B$. Therefore we get

$$
\operatorname{dim} X-2 \geq \operatorname{dim} B \geq \operatorname{dim} \operatorname{ChLocus}(\mathcal{V})_{F} \geq f_{V}+m+2 \geq \operatorname{dim} X-1,
$$

which is a contradiction.

As the last preparatory step, we consider the following special case.

Lemma 4.2. Let $V$ be a quasi-unsplit dominating family of rational curves on a smooth complex projective variety $X$. Denote by $f_{V}$ the dimension of a general $r c(\mathcal{V})$-equivalence class. Assume that there exists an extremal ray $\vartheta$, independent from $[V]$, whose associated contraction has a fiber $F$ such that $\operatorname{dim} F+f_{V} \geq \operatorname{dim} X$. Then $\operatorname{dim} F+f_{V}=\operatorname{dim} X$ and $\operatorname{NE}(X)=\langle[V], \vartheta\rangle_{c}$. In particular $\rho_{X}=2$.

Proof. By part (1) of Lemma 2.4 we have

$$
\operatorname{dim} X \geq \operatorname{dim} \operatorname{ChLocus}(\mathcal{V})_{F} \geq f_{V}+\operatorname{dim} F,
$$

hence $\operatorname{dim} F+f_{V}=\operatorname{dim} X$ and $\operatorname{ChLocus}(\mathcal{V})_{F}=X$; so the assertion follows by Corollary (1.11).

Theorem 4.3. Let $(X, H)$ be a polarized manifold with a dominating family of rational curves $V$ such that $H \cdot V=1$. If $-K_{X} \cdot V \geq \frac{\operatorname{dim} X-1}{2}$, then $[V]$ spans an extremal ray of $\mathrm{NE}(X)$.

Proof. Let $B$ be the indeterminacy locus of the $\operatorname{rc}(\mathcal{V})$-fibration $q: X-->Y$, let $D$ be a very ample divisor on $q(X \backslash B)$ and let $\widehat{D}:=\overline{q^{-1} D}$. Denote by $m$ the anticanonical degree of $V$ and by $f_{V}$ the dimension of a general $\operatorname{rc}(\mathcal{V})$-equivalence class. Notice that, since $V$ is a dominating family, we have $m \geq 2$. 
By Proposition $1.2 \operatorname{dim} \operatorname{Locus}\left(V_{x}\right) \geq-K_{X} \cdot V-1=m-1$; since a general fiber of the $\operatorname{rc}(\mathcal{V})$-fibration contains $\operatorname{Locus}\left(V_{x}\right)$ for every point $x$ in it, we have $f_{V} \geq m-1$.

If $K_{X}+m H$ is nef, then the assertion follows by Proposition (4.1); therefore we can assume that $K_{X}+m H$ is not nef.

Let $\vartheta$ be an extremal ray such that $\left(K_{X}+m H\right) \cdot \vartheta<0$ and let $\varphi_{\vartheta}$ be the associated contraction. Notice that $\vartheta$ has length $\ell(\vartheta) \geq m+1$, hence every non-trivial fiber of $\varphi_{\vartheta}$ has dimension $\geq m$ by Proposition (1.3). On the other hand, by Lemma (4.2) we can confine to assume that all fibers of $\varphi_{\vartheta}$ have dimension $\leq m+1$.

In particular this implies that, denoted by $C_{\vartheta}$ a minimal degree curve whose numerical class belongs to $\vartheta$, we have $H \cdot C_{\vartheta}=1$. Indeed, if this were not the case, we would have $\ell(\vartheta) \geq 2 m+1$, hence every non-trivial fiber of $\varphi_{\vartheta}$ would have dimension $\geq 2 m>m+1$, by Proposition (1.3) and the fact that $m \geq 2$.

If the Picard number of $X$ is one the theorem is clearly true, so we can assume that $\rho_{X} \geq 2$. Now we split up the proof in two cases, according to the value of $\rho_{X}$ : first we consider the case $\rho_{X}=2$ and then the general one.

Case (a) $\rho_{X}=2$.

The proof is based on different arguments, depending on the dimension of the fibers of the contraction associated to the extremal ray $\vartheta$.

Case (a1) The contraction $\varphi_{\vartheta}$ admits an $(m+1)$-dimensional fiber $F$.

Consider $X_{F}:=\operatorname{ChLocus}(\mathcal{V})_{F}$. We have, by Corollary (1.11), that NE $\left(X_{F}, X\right)=$ $\langle[V], \vartheta\rangle_{c}$ and, by Lemma (2.4), that

$$
\operatorname{dim} X_{F} \geq \operatorname{dim} F+f_{V} \geq(m+1)+(m-1) \geq \operatorname{dim} X-1 .
$$

If $X_{F}=X$, then the statement is proved. So we can assume that an irreducible component $\bar{X}_{F}$ of $X_{F}$ is a divisor and thus that $f_{V}=m-1$. Notice that $\bar{X}_{F} \cdot V=0$, otherwise we would have $X_{F}=X$.

Consider now the intersection number of $X_{F}$ with curves whose numerical class belongs to $\vartheta$; since $\rho_{X}=2$ and $\bar{X}_{F} \cdot V=0$ we cannot have also $\bar{X}_{F} \cdot \vartheta=0$.

Let us show that we cannot have $\bar{X}_{F} \cdot \vartheta<0$, too.

Assume by contradiction that this is the case. Then $\operatorname{Exc}(\vartheta) \subset \bar{X}_{F}$, so $\varphi_{\vartheta}$ is divisorial by Proposition (1.3). By the same proposition, recalling that we are assuming that all the fibers of $\varphi_{\vartheta}$ have dimension $\leq m+1$, every non-trivial fiber has dimension $m+1$.

Then $\varphi_{\vartheta}$ is the blow-up of a smooth variety $X^{\prime}$ along a smooth center $T$ by 2 , Theorem 4.1 (iii)]. The dimension of the center is

$$
\operatorname{dim} T=(n-1)-(m+1) \leq m-1=f_{V} .
$$

We can thus apply part (4) of Lemma (3.1) and we get $\rho_{X}=\rho_{X^{\prime}}+1>2$, reaching a contradiction.

Therefore $\bar{X}_{F} \cdot \vartheta>0$, hence $\left.\left(\bar{X}_{F}\right)\right|_{\bar{X}_{F}}$ is nef and thus, by Lemma 1.12, $\bar{X}_{F}$ is nef. As $\bar{X}_{F} \cdot V=0$ and $\rho_{X}=2, \bar{X}_{F}$ is the supporting divisor of an elementary contraction of $X$ whose associated extremal ray is spanned by $[V]$.

Case (a2) The contraction $\varphi_{\vartheta}$ is equidimensional with $m$-dimensional fibers.

By Proposition (1.3), $\varphi_{\vartheta}$ is of fiber type and $\ell(\vartheta)=m+1$. Hence, by [11, Lemma 2.12], $X$ is a projective bundle over a smooth variety $Y$, i.e. $X=\mathbb{P}_{Y}(\mathcal{E})$, where $\mathcal{E}=\left(\varphi_{\vartheta}\right)_{*} H$. 
Notice that $Y$ has Picard number one and is covered by rational curves - the images of the curves parametrized by $V$ - therefore $Y$ is a Fano manifold.

By the canonical bundle formula for projective bundles we have

$$
K_{X}+(m+1) H=\varphi_{\vartheta}^{*}\left(K_{Y}+\operatorname{det} \mathcal{E}\right) .
$$

In particular, if $C_{V}$ is a curve among those parametrized by $V$, by the projection formula we can compute

$$
\left(K_{Y}+\operatorname{det} \mathcal{E}\right) \cdot\left(\varphi_{\vartheta}\right)_{*}\left(C_{V}\right)=\left(K_{X}+(m+1) H\right) \cdot C_{V}=1 .
$$

It follows that $\left(K_{Y}+\operatorname{det} \mathcal{E}\right) \cdot \varphi_{\vartheta}\left(C_{V}\right)=1$ and that $K_{Y}+\operatorname{det} \mathcal{E}$ is the ample generator of $\operatorname{Pic}(Y)$. The ampleness of $\mathcal{E}$ implies that $\operatorname{det} \mathcal{E} \cdot \varphi_{\vartheta}\left(C_{V}\right) \geq m+1$; therefore $-K_{Y} \cdot \varphi_{\vartheta}\left(C_{V}\right) \geq m$, hence the index $r_{Y}$ of $Y$ is greater than or equal to $m$.

If $r_{Y}=m$, denoted by $l$ a rational curve of minimal degree in $Y$, then $\operatorname{det} \mathcal{E} \cdot l=$ $m+1$; moreover, the splitting type of $\mathcal{E}$, which is ample and of rank $m+1$, on rational curves of minimal degree is uniform of type $(1, \ldots, 1)$.

We can thus apply [3, Proposition 1.2], so we obtain that $X \simeq \mathbb{P}^{m} \times Y$. It follows that the curves of $V$ are contained in the fibers of the first projection and that $[V]$ spans an extremal ray.

Therefore we are left with $r_{Y} \geq m+1$. Recalling that $\operatorname{dim} Y=\operatorname{dim} X-m \leq$ $m+1$, by the Kobayashi-Ochiai Theorem ([13]) we get that $Y$ is a projective space or a hyperquadric.

Assume by contradiction that $[V]$ does not span an extremal ray of $X$.

By part (3) of Lemma 2.2 there exists a curve $C \subset B$, whose numerical class is not proportional to $[V]$, such that $\widehat{D} \cdot C \leq 0$. Actually, since $\rho_{X}=2$ and $\widehat{D} \cdot V=0$, we have $\widehat{D} \cdot C<0$.

By part (2) of Lemma 2.4 , there exists $X_{C} \subset \operatorname{ChLocus}(\mathcal{V})_{C}$ which is not $\operatorname{rc}(\mathcal{V})$ connected such that $\operatorname{dim} X_{C} \geq f_{V}+\operatorname{dim} C+1 \geq m+1$.

By Lemma 1.10 $\widehat{D}$ has non positive intersection number with every curve in $X_{C}$ and it is trivial only on curves which are numerically proportional to $[V]$.

Since $\widehat{D} \cdot \vartheta>0$, we have that $\varphi_{\vartheta}$ does not contract curves in $X_{C}$, hence $\operatorname{dim} Y \geq$ $\operatorname{dim} X_{C} \geq m+1$ and so $\operatorname{dim} Y=\operatorname{dim} X_{C}=m+1$.

Since $X_{C}$ is not $\operatorname{rc}(\mathcal{V})$-connected, for every point $c$ of $X_{C}$, the intersection $X_{c}$ of the $\operatorname{rc}(\mathcal{V})$-equivalence class containing $c$ with $X_{C}$ has dimension $=m$. In particular $X_{C}$ is the union of a one parameter family of $\operatorname{rc}(\mathcal{V})$-connected subvarieties $X_{c}$.

We claim that there exists a line $l$ in $Y$ which is not contained in $\varphi_{\vartheta}\left(X_{c}\right)$ for any $c \in C$. Notice that, since $\varphi_{\vartheta}$ does not contract curves in $X_{C}$, through a general point $y$ in $Y$ there is a finite number of such subvarieties.

If $Y \simeq \mathbb{P}^{m+1}$, a line joining $y$ with a point outside the union of these subvarieties has the required property.

Assume now that $Y \simeq \mathbb{Q}^{m+1}$; for any $y \in \mathbb{Q}^{m+1}$ the locus of the lines through $y$ is a quadric cone $\mathbb{Q}_{y}^{m}$ with vertex $y$. Therefore, if every line through $y$ is contained in $\varphi_{\vartheta}\left(X_{c}\right)$ for some $c \in C$, then $\mathbb{Q}_{y}^{m}$ is an irreducible component of $\varphi_{\vartheta}\left(X_{c}\right)$; since $X_{c}$ moves in a one-dimensional family, for the general point $y \in \mathbb{Q}^{m+1}$, the general line through $y$ has the required property.

The splitting type of $\mathcal{E}$ on this line is one of the following: $(2,1, \ldots, 1)$ if $Y \simeq$ $\mathbb{Q}^{m+1}$ and either $(3,1, \ldots, 1)$ or $(2,2,1, \ldots, 1)$ if $Y \simeq \mathbb{P}^{m+1}$. Recalling that $m \geq 2$ we have that, among the summands of $\mathcal{E}_{l}$ there is at least one $\mathcal{O}_{\mathbb{P}^{1}}(1)$. 
Consider $\mathbb{P}_{l}\left(\left.\mathcal{E}\right|_{l}\right)$; its cone of curves is generated by the class of a line in a fiber of the projection onto $l$ and the class of a minimal section $C_{0}$. By the discussion above we have that $H \cdot C_{0}=1$. Moreover, $\varphi_{\vartheta}^{*}\left(K_{Y}+\operatorname{det} \mathcal{E}\right) \cdot C_{0}=1$, hence $\left[C_{0}\right]=[V]$; in particular $\widehat{D}$ is nef on $\mathbb{P}_{l}\left(\left.\mathcal{E}\right|_{l}\right)$.

Consider an irreducible curve in $\mathbb{P}_{l}\left(\left.\mathcal{E}\right|_{l}\right) \cap X_{C}$; by our choice of $l$, this curve is not contained in $\operatorname{arc}(\mathcal{V})$-equivalence class contained in $X_{C}$, so it is negative with respect to $\widehat{D}$, a contradiction. The case $\rho_{X}=2$ is thus completed.

Case (b) $\rho_{X}>2$.

Notice that, in view of Corollary $(2.3)$, we can confine to assume that $B \neq \emptyset$; moreover, by part (3) of Lemma 2.2 , we can also assume the existence of a curve $C \subset B$ such that $[C]$ is not proportional to $[V]$ and $\widehat{D} \cdot C \leq 0$.

We claim that $K_{X}+(m+1) H$ is nef.

Assume by contradiction that $K_{X}+(m+1) H$ is not nef. Let $\tau$ be a ray such that $\left(K_{X}+(m+1) H\right) \cdot \tau<0$, denote by $C_{\tau}$ a rational curve of minimal anticanonical degree in $\tau$ and by $\varphi_{\tau}$ the contraction associated to $\tau$.

Notice that $\tau$ has length $\ell(\tau) \geq m+2$, hence every non-trivial fiber of $\varphi_{\tau}$ has dimension $\geq m+1$ by Proposition (1.3).

On the other hand $\varphi_{\tau}$ cannot have fibers of dimension $>m+1$, otherwise, by Lemma 4.2 , we would have $\rho_{X}=2$. Therefore every non-trivial fiber of $\varphi_{\tau}$ has dimension $m+1$.

In view of Proposition (1.3), we thus get that $\varphi_{\tau}$ is of fiber type and that the length of $\tau$ is $\ell(\tau)=m+2$; this last fact gives $H \cdot C_{\tau}=1$. Let us consider $W_{\tau}$ to be a minimal degree covering family of curves whose numerical class belongs to $\tau$.

Since $B$ is not empty, there $\operatorname{are~} \operatorname{rc}(\mathcal{V})$-equivalence classes of dimension $\geq f_{V}+1 \geq m$; let $G$ be one of these classes. Notice that since $\varphi_{\tau}$ is equidimensional with $(m+1)$ dimensional fibers, we have $f_{W}=m+1$. By part (1) of Lemma (2.4) we have

$$
\operatorname{dim} \operatorname{ChLocus}\left(\mathcal{W}_{\tau}\right)_{G} \geq \operatorname{dim} G+f_{W}=2 m+1 \geq \operatorname{dim} X,
$$

so by Lemma $(1.9)$ we deduce $\rho_{X}=2$, a contradiction which proves the nefness of $K_{X}+(m+1) H$.

Recall now that the extremal ray $\vartheta$ which we fixed at the beginning of the proof has length $\ell(\vartheta) \geq m+1$ and is generated by a curve $C_{\vartheta}$ such that $H \cdot \vartheta=1$, therefore $\left(K_{X}+(m+1) H\right) \cdot \vartheta=0$ and $K_{X}+(m+1) H$ is not ample.

Let $\Sigma$ be the extremal face contracted by $K_{X}+(m+1) H$. We now consider separately two cases, depending on the existence in $\Sigma$ of a fiber type extremal ray.

Case (b1) There exists a fiber type extremal ray $\varrho$ in $\Sigma$.

Let $\varphi_{\varrho}$ be the contraction associated with $\varrho$ and denote by $W_{\varrho}$ a minimal degree covering family of curves whose numerical class belongs to $\varrho$.

By part (2) of Lemma (2.4), there exists an irreducible $X_{C} \subset \operatorname{ChLocus}(\mathcal{V})_{C}$ such that $\operatorname{dim} X_{C} \geq f_{V}+2$.

According to Lemma 1.10 , every curve in $X_{C}$ can be written as $\alpha[C]+\beta[V]$ with $\alpha \geq 0$; in particular, since $\widehat{D} \cdot V=0$ by Lemma 2.2 , it follows that $\widehat{D}$ is not positive on any curve contained in $X_{C}$. By the same lemma $\widehat{D} \cdot W_{\varrho}>0$, hence $\left[W_{\varrho}\right] \notin \mathrm{NE}\left(X_{C}, X\right)$. Therefore part (1) of Lemma (2.4) gives

$$
\operatorname{dim} \operatorname{ChLocus}\left(\mathcal{W}_{\varrho}\right)_{X_{C}} \geq \operatorname{dim} X_{C}+f_{W_{\varrho}} \geq f_{V}+2+m \geq \operatorname{dim} X,
$$


where $f_{W_{\varrho}}$ is the dimension of the general $\operatorname{rc}\left(\mathcal{W}_{\varrho}\right)$-equivalence class.

Therefore, by applying twice Lemma 1.10, we get that the class of every curve in $X$ can be written as

$$
\lambda(\alpha[C]+\beta[V])+\mu\left[W_{\varrho}\right]
$$

with $\alpha, \lambda \geq 0$ and $\alpha[C]+\beta[V] \in \mathrm{NE}\left(X_{C}, X\right)$.

This has some very important consequences: first of all, since we are assuming $\rho_{X}>2$, this implies that $\rho_{X}=3$; in particular $[C]$ is not contained in the plane $\Pi$ in $\mathrm{N}_{1}(X)$ spanned by $\left[W_{\varrho}\right]$ and $[V]$. Moreover the intersection of $\Pi$ with $\mathrm{NE}(X)$ is a face of $\mathrm{NE}(X)$.

We have to prove that $\Pi \cap \overline{\mathrm{NE}}(X)=\left\langle[V],\left[W_{\varrho}\right]\right\rangle_{c}$. If this is not the case, then there exists a class $a$ such that $\Pi \cap \overline{\mathrm{NE}}(X)=\left\langle a,\left[W_{\varrho}\right]\right\rangle_{c}$ and $\widehat{D} \cdot a<0$.

Denote by $b \in \mathrm{N}_{1}(X)$ a class, not proportional to $[V]$, lying in the intersection of $\partial \overline{\mathrm{NE}}(X)$ with the plane $\Pi^{\prime}=\mathrm{N}_{1}\left(X_{C}, X\right)$ and by $\Pi^{\prime \prime}$ the plane spanned by $\left[W_{\varrho}\right]$ and $b$.

Formula 4.3.1), traslated in geometric terms, says that $\mathrm{NE}(X)$ is contained in the intersection of half-spaces determined by $\Pi$ and by $\Pi^{\prime \prime}$ as in the figure below, which shows a cross-section of $\overline{\mathrm{NE}}(X)$.

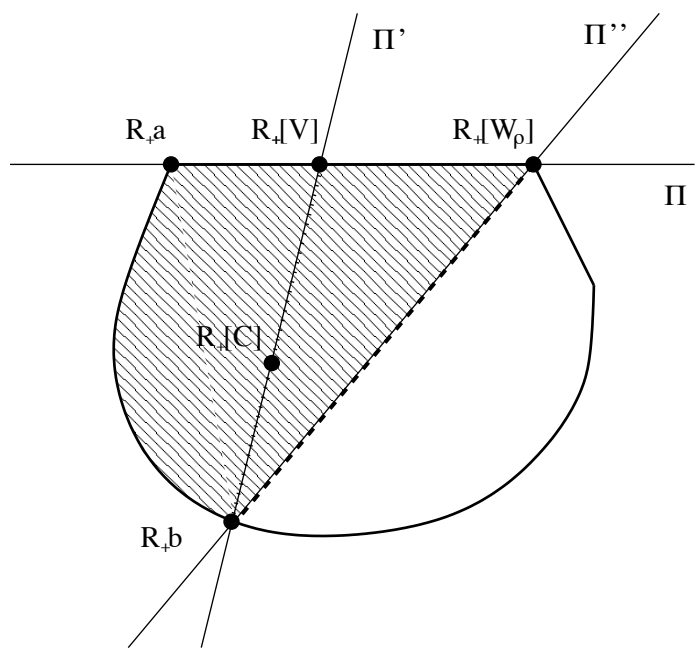

Let $\left\{C_{n}\right\}$ be a sequence of effective one cycles such that the limit of $\mathbb{R}_{+}\left[C_{n}\right]$ is $\mathbb{R}_{+} a$; by continuity, for some $n_{0}$ we have $\widehat{D} \cdot C_{n}<0$ for $n \geq n_{0}$, hence $C_{n} \subset B$ for $n \geq n_{0}$, and all the above arguments apply to $C_{n}$, for $n \geq n_{0}$. In particular, defining $b_{n}$ and $\Pi_{n}^{\prime \prime}$ as above, we get that, for $n \geq n_{0}, \mathrm{NE}(X)$ is contained in the intersection of half-spaces determined by $\Pi$ and by $\Pi_{n}^{\prime \prime}$. Since $\Pi_{n}^{\prime \prime} \rightarrow \Pi$ as $\mathbb{R}_{+}\left[C_{n}\right] \rightarrow \mathbb{R}_{+} a$ and $\rho_{X}=3$ we get a contradiction.

Case (b2) Every ray in $\Sigma$ is birational.

Let $\eta$ be any ray in $\Sigma$. By Proposition (1.3), for every non-trivial fiber of its associated contraction $\varphi_{\eta}$ we have $\operatorname{dim} F \geq \ell(\eta) \geq m+1$. Recalling that, by Lemma 4.2, we can assume $\operatorname{dim} F \leq m+1$, we have $\operatorname{dim} F=m+1=\ell(\eta)$. This also implies that, if $C_{\eta}$ is a minimal degree curve whose numerical class is contained in $\eta$ we have $H \cdot C_{\eta}=1$.

By Proposition 1.3$], \varphi_{\eta}$ is a divisorial contraction, hence, by [2, Theorem 4.1 
(iii)], is the blow-up of a smooth variety along a smooth center $T$ of dimension $(n-1)-(m+1) \leq m-1$.

Let $E$ be the exceptional divisor of $\varphi_{\eta}$. By part (2) of Lemma (2.4), there exists an irreducible $X_{C} \subset \operatorname{ChLocus}(\mathcal{V})_{C}$ with $\operatorname{dim} X_{C} \geq f_{V}+2$.

By Lemma $1.10 \widehat{D}$ has non positive intersection number with every curve in $X_{C}$. If $E \cap X_{C} \neq \emptyset$, then there is a fiber $F$ of $\varphi_{\eta}$ meeting $X_{C}$. Counting dimensions, we find that $\operatorname{dim}\left(F \cap X_{C}\right) \geq 1$, which is a contradiction as $\widehat{D} \cdot \eta>0$. So $E \cap X_{C}=\emptyset$, whence $E \cdot V=0$.

Therefore $E$ contains $\operatorname{rc}(\mathcal{V})$-equivalence classes and $\operatorname{dim} T \geq f_{V}$, since $\varphi_{\eta}$ is finiteto-one on $\operatorname{rc}(\mathcal{V})$-equivalence classes. Recalling that $f_{V} \geq m-1$ we derive $\operatorname{dim} T=$ $f_{V}=m-1$.

Assume that $\operatorname{dim} \Sigma \geq 2$ and let $E_{1}, E_{2}$ be the exceptional loci of two different extremal rays $\eta_{1}, \eta_{2}$ in $\Sigma$; since the fibers of the contractions $\varphi_{\eta_{1}}$ and $\varphi_{\eta_{2}}$ have dimension $m+1$ and $2(m+1)>\operatorname{dim} X$ we have that $E_{1} \cap E_{2}=\emptyset$.

Therefore the contraction $\sigma: X \rightarrow X^{\prime}$ of the face $\Sigma$ verifies the assumptions of Lemma (3.1), hence there exists an ample line bundle $H^{\prime}$ on $X^{\prime}$ and an unsplit dominating family $V^{\prime}$ on $X^{\prime}$ such that $H^{\prime} \cdot V^{\prime}=1$ and $-K_{X^{\prime}} \cdot V^{\prime}=-K_{X} \cdot V \geq$ $\frac{\operatorname{dim} X^{\prime}-1}{2}$.

Denote by $f_{V^{\prime}}$ the dimension of the general $\mathrm{rc}\left(\mathcal{V}^{\prime}\right)$-equivalence class. Since a general fiber of the $\operatorname{rc}\left(\mathcal{V}^{\prime}\right)$-fibration contains $\operatorname{Locus}\left(V_{x^{\prime}}^{\prime}\right)$, we have $f_{V^{\prime}} \geq \operatorname{dim} \operatorname{Locus}\left(V_{x^{\prime}}^{\prime}\right)-1 \geq$ $m-1$.

Consider the adjoint divisor $K_{X^{\prime}}+m H^{\prime}$; if it is nef, or an extremal ray $\vartheta^{\prime}$ such that $\left(K_{X^{\prime}}+m H^{\prime}\right) \cdot \vartheta^{\prime}<0$ has a fiber of dimension $\geq m+2$, then $\left[V^{\prime}\right]$ spans an extremal ray by Proposition (4.1) or by Lemma (4.2), so $[V]$ spans an extremal ray by Lemma (3.1).

Let us show that the remaining case does not happen.

Assume that there is an extremal ray $\vartheta^{\prime}$ such that $\left(K_{X^{\prime}}+m H^{\prime}\right) \cdot \vartheta^{\prime}<0$ and every fiber of the associated contraction has dimension $\leq m+1$. In particular we have $H^{\prime} \cdot \vartheta^{\prime}=1$, otherwise we would have $\ell\left(\vartheta^{\prime}\right) \geq 2 m+1$, hence every non-trivial fiber of the associated contraction would have dimension $\geq 2 m>m+1$ by Proposition (1.3). Moreover, we have $\left(K_{X^{\prime}}+(m+1) H^{\prime}\right) \cdot \vartheta^{\prime} \leq 0$, since $\ell\left(\vartheta^{\prime}\right) \geq m+1$.

On the other hand, recalling that $\sigma^{*} H^{\prime}=H+\sum E_{i}$ and that $\sigma^{*} K_{X^{\prime}}=K_{X}-$ $\sum(m+1) E_{i}$, we have

$$
\sigma^{*}\left(K_{X^{\prime}}+(m+1) H^{\prime}\right)=K_{X}+(m+1) H
$$

so, by the projection formula, $K_{X^{\prime}}+(m+1) H^{\prime}$ is ample on $X^{\prime}$, a contradiction.

Corollary 4.4. Let $(X, H)$ be a polarized manifold of dimension at most five, with a dominating family of rational curves $V$ such that $H \cdot V=1$. Then $[V]$ spans an extremal ray of $\mathrm{NE}(X)$.

\section{An EXAmple}

In the paper [5], an application of the results about extremality of families of lines was a relative version of a theorem proved in [18, which was the first step towards a conjecture of Mukai for Fano manifolds.

This conjecture states that, for a Fano manifold $X$, denoted by $\rho_{X}$ its Picard 
number and by $r_{X}$ its index, we have

$$
\rho_{X}\left(r_{X}-1\right) \leq \operatorname{dim} X
$$

More precisely, in [18, Theorem B] it was proved that, if $r_{X} \geq \frac{\operatorname{dim} X}{2}+1$, then $\rho_{X}=1$ unless $X \simeq \mathbb{P}^{\operatorname{dim} X / 2} \times \mathbb{P}^{\operatorname{dim} X / 2}$, while in [5, Theorem 3.1.1] it was proved that a fiber type contraction $\varphi: X \rightarrow Y$ supported by $K_{X}+m L$ with $m \geq \frac{\operatorname{dim} X}{2}+1$ is elementary, unless $X \simeq \mathbb{P}^{\operatorname{dim} X / 2} \times \mathbb{P}^{\operatorname{dim} X / 2}$.

In the last few years some progress has been made towards Mukai conjecture; in particular it was recently proved in [16, Theorem 3] that it holds for a Fano manifold with (pseudo)index greater than or equal to $\frac{\operatorname{dim} X}{3}+1$.

It is therefore natural to ask if the corresponding relative statement is true, namely, given a fiber type contraction $\varphi: X \rightarrow Y$, corresponding to an extremal face $\Sigma$, supported by $K_{X}+m L$ with $m \geq \frac{\operatorname{dim} X}{3}+1$ is it possible to find a bound on the dimension of $\Sigma$ ?

The answer to this question is negative, as we will show with an example in which $m=\frac{\operatorname{dim} X}{2}$; it follows that [5, Theorem 3.1.1] cannot be improved.

Example 5.1. Let $Z$ be a smooth variety of dimension $k+2$, denote by $Y$ the product $Z \times \mathbb{P}^{k}$ and by $p_{1}, p_{2}$ the projections onto the factors. Let $\left\{z_{i}\right\}_{i=1, \ldots, t}$ be points of $Z$ and denote by $F_{i}$ the fibers of $p_{1}$ over $z_{i}$.

Let $\sigma: X \rightarrow Y$ be the blow-up of $Y$ along the union of $F_{i}$. The canonical bundle of $X$ is

$$
K_{X}=\sigma^{*} K_{Y}+(k+1) \sum_{i=1}^{t} E_{i}=\sigma^{*}\left(p_{1}^{*} K_{Z}+p_{2}^{*} K_{\mathbb{P}^{k}}\right)+(k+1) \sum_{i=1}^{t} E_{i} ;
$$

denoting by $\mathcal{H}:=\left(p_{2} \circ \sigma\right)^{*} \mathcal{O}_{\mathbb{P}^{k}}(1)$ and by $L^{\prime}:=\mathcal{H}-\sum E_{i}$, we can rewrite formula 5.1.1 as

$$
K_{X}+(k+1) L^{\prime}=\sigma^{*}\left(p_{1}^{*} K_{Z}\right) .
$$

It is easy to check that $L^{\prime}$ is $\left(p_{1} \circ \sigma\right)$-ample. Let $A \in \operatorname{Pic}(Z)$ be an ample line bundle such that $K_{Z}+(k+1) A$ is ample; then $L:=L^{\prime}+\sigma^{*}\left(p_{1}^{*} A\right)$ is an ample line bundle on $X$; moreover $L \cdot l=1$ for a line $l$ in the strict transform of a fiber $F$ of $p_{1}$ not contained in the center of $\sigma$.

The contraction $p_{1} \circ \sigma$ is supported by $K_{X}+(k+1) L=K_{X}+\frac{\operatorname{dim} X}{2} L$ and contracts a face of dimension $t+1$.

Remark 5.2. The difference between the relative and the absolute case is given by the existence of minimal horizontal dominating families of rational curves for proper morphisms defined on a open subset of a Fano manifold (for the definition and the references see [1, Remark 6.4]). Such families do not exist in general in the relative case.

\section{ACKNOWLEDGEMENTS}

We learned of the results about extremality of families of lines in [5] from an interesting series of lectures given by Paltin Ionescu. We thank the referee for many useful suggestions and remarks, which helped to fix some issues in the proofs. 


\section{REFERENCES}

[1] Marco Andreatta, Elena Chierici, and Gianluca Occhetta. Generalized Mukai conjecture for special Fano varieties. Cent. Eur. J. Math., 2(2):272-293, 2004.

[2] Marco Andreatta and Jarosław A. Wiśniewski. A note on nonvanishing and applications. Duke Math. J., 72(3):739-755, 1993.

[3] Marco Andreatta and Jarosław A. Wiśniewski. On manifolds whose tangent bundle contains an ample subbundle. Invent. Math., 146(1):209-217, 2001.

[4] Mauro C. Beltrametti and Paltin Ionescu. On manifolds swept out by high dimensional quadrics Math. Z., 260(1):229-236, 2008.

[5] Mauro C. Beltrametti, Andrew J. Sommese, and Jarosław A. Wiśniewski. Results on varieties with many lines and their applications to adjunction theory. In Complex algebraic varieties (Bayreuth, 1990), volume 1507 of Lecture Notes in Math., pages 16-38. Springer, Berlin, 1992.

[6] Laurent Bonavero, Cinzia Casagrande, and Stéphane Druel. On covering and quasi-unsplit families of rational curves. J. Eur. Math. Soc., 9(1):45-57, 2007.

[7] F. Campana. Coréduction algébrique d'un espace analytique faiblement kählérien compact. Invent. Math., 63(2):187-223, 1981.

[8] F. Campana. Connexité rationnelle des variétés de Fano. Ann. Sci. École Norm. Sup. (4), 25(5):539-545, 1992.

[9] Elena Chierici and Gianluca Occhetta. The cone of curves of Fano varieties of coindex four. Internat. J. Math., 17(10):1195-1221, 2006.

[10] Olivier Debarre. Higher-dimensional algebraic geometry. Universitext. Springer-Verlag, New York, 2001.

[11] Takao Fujita. On polarized manifolds whose adjoint bundles are not semipositive. In Algebraic geometry, Sendai, 1985, volume 10 of Adv. Stud. Pure Math., pages 167-178. North-Holland, Amsterdam, 1987.

[12] Paltin Ionescu. Generalized adjunction and applications. Math. Proc. Cambridge Philos. Soc., 99(3):457-472, 1986.

[13] Shoshichi Kobayashi and Takushiro Ochiai. Characterizations of complex projective spaces and hyperquadrics. J. Math. Kyoto Univ., 13:31-47, 1973.

[14] János Kollár. Rational curves on algebraic varieties, volume 32 of Ergebnisse der Mathematik und ihrer Grenzgebiete. Springer-Verlag, Berlin, 1996.

[15] Carla Novelli and Gianluca Occhetta. Projective manifolds containing a large linear subspace with nef normal bundle. Michigan Mathematical Journal, to appear.

[16] Carla Novelli and Gianluca Occhetta. Rational curves and bounds on the Picard number of Fano manifolds. Geometriae Dedicata, 147:207-217, 2010.

[17] Gianluca Occhetta. A characterization of products of projective spaces. Canad. Math. Bull., 49:270-280, 2006.

[18] Jarosław A. Wiśniewski. On a conjecture of Mukai. Manuscripta Math., 68(2):135-141, 1990.

[19] Jarosław A. Wiśniewski. On contractions of extremal rays of Fano manifolds. J. Reine Angew. Math., 417:141-157, 1991.

Dipartimento di Matematica "F. Casorati",

Università di PAVIA,

VIA FerRata 1,

I-27100 PAVIA

Current address: Dipartimento di Matematica e Applicazioni,

Università di Milano - Bicocca,

via R. Cozzi 53,

I-20126 Milano

E-mail address: carla.novelli@unimib.it

Dipartimento di Matematica,

Università di TRENTO,

VIa Sommarive 14,

I-38050 Povo (TN)

E-mail address: gianluca.occhetta@unitn.it 